\title{
Sweet and Bitter Tastes Evoked Different Neuronal Activation in the Rostral Portion of the Nucleus of the Solitary Tract of Developing Rats*
}

\author{
Lorena Rubio-Navarro, Carmen Torrero, Mirelta Regalado, Manuel Salas \\ Departamento de Neurobiología del Desarrollo y Neurofisiología, Instituto de Neurobiología, \\ Universidad Nacional Autónoma de México, Querétaro, México \\ Email:masal@unam.mx
}

Received March 14, 2012; revised April 18, 2012; accepted April 30, 2012

\begin{abstract}
The impact of the gustatory stimuli on the rostral portion of the nucleus of the solitary tract (rNST) was investigated in developing rats, by using c-fos immunohistochemical staining. Wistar male rats of 5,15 , and 25 days of age were isolated from the mother for $12 \mathrm{~h}$, then stimulated via the intraoral route with quinine, sucrose, or $\mathrm{NaCl}$, and sacrificed 90 min later. The water-stimulated group showed minimal c-fos-like immunoreactivity (FLI) compared with taste-stimulated groups that exhibited different FLI in the rNST at the different ages. At all ages the quinine-stimulated group induced FLI in the medial subfield, while sucrose induced FLI in the lateral subfield of the rNST. The intensity of FLI was highest at P15, and it declined at P25. These findings provide detailed insight into the anatomical basis of rNST activation that is involved in early food intake and the learning capacity of the newborn.
\end{abstract}

Keywords: c-fos; Rostral Portion of the Nucleus of the Solitary Tract; Gustatory Development; Rats

\section{Introduction}

In altricial newborn mammals the gustatory system provides the anatomical substrate for the selective ingestion or rejection of nutrients and for the establishment of strong links with environmental nest cues to gain olfactory and gustatory experience needed for survival $[1,2]$. The complete gustatory receptors, their afferent projecttions, and the neurons in the rNST, which is the first relay in the gustatory pathway, are far from fully developed at birth [3-7]. Neurons in the rNST undergo distinctive qualitative and quantitative changes during the first 30 days after birth [8-10]. Electrophysiological studies of the rat rNST show changes in the intrinsic membrane properties of its neurons between early development and the adult stage [11-13]. At the single-cell level, an analysis of the neurons that respond to gustatory cues shows they are functional by the end of the first postnatal week [14].

The organization of the rNST in adult mammals has been studied previously [15-17], but at present little information is available on the development of this structure in the rat [10]. Analysis of the gustofacial responses of newly born rats to different tastes indicates that from the first day of life, pups respond to quinine solution with

*The authors declare that there are no conflicts of interests concerning this research. certain aversive characteristics (gaping and forelimb flailing) that suppress oral intake [10]. Other components of the adult-like aversive response (chin scraping and paw treading) do not appear until 12 days of age. This can be due to the pup's inability to detect aversive solutions, because of the neuronal substrate immaturity subserving these motor behavioral responses $[18,19]$. In the case of sucrose, a single droplet into the mouth of the newborn elicits licking and rhythmic mouth movements as the most salient feature [20]. The entire set of behavioral and discriminatory components underlying the different responsiveness to sweet and bitter tastes in the rat emerge over the first 2 weeks of life [18-21].

The expression of the immediate-early gene c-fos, detected by the immunohistochemical c-fos protein staining technique, has been used as an anatomical marker of activated neurons in the central nervous system [22]. Previous studies have shown in adult rats that gustatory stimulation induces c-fos-like immunoreactivity (FLI) in NST neurons, and they are activated differentially by sucrose (S) and quinine monohydrochloride $(\mathrm{QHCl})$, two taste cues that differ importantly in qualitative, hedonic, and behavioral characteristics [15-17]. Thus, the sucrose stimulation elicits FLI in neurons distributed along the mediolateral axis of the rNST; in contrast, the stimulation by QHCl exhibits a prominent, but more medial FLI in 
the rNST [15-17]. These differences in the distribution of stimulus properties appear to be a ubiquitous feature of the sensory systems. The most obvious topography in the gustatory system is the systematic representation of information arising from taste buds in different parts of the mouth. This organization is best documented in the NST, where it appears to be a rostral continuation of a topographic representation of the entire gastrointestinal tract [15-17,23-25]. The neural areas that are activated early in life by gustatory stimulation have not been previously analyzed. The current experiment was designed to address if the anatomical substrate underlying the sucrose and quinine gustatory responses is already functional during the first days of life by evaluating c-fos immunoreactivity in response to different taste stimuli in rNST neurons.

\section{Materials and Methods}

\subsection{Animals}

Male Wistar rats (Rattus norvegicus), descendants of a stock originally purchased from Harlan Sprague-Dawley, Indianapolis (IN), and subsequently bred from our laboratory stock at the Institute of Neurobiology University of Mexico; at 5, 15 and 25 days of age were used. Litter size ( $\mathrm{n}=8$ pups, 4 males and 4 females/age) were maintained under a 12:12 h light-dark cycle (lights on at 08:00 h) in a room at $23^{\circ} \mathrm{C} \pm 2^{\circ} \mathrm{C}$ with humidity of about $60 \%$, and were housed with mothers, who had free access to food (Purina chow) and water. Animals were handled in accordance with the National Institutes of Health Guide for the Care and Use of Laboratory Animals. All efforts were made to use a minimal number of animals in the experiments.

\subsection{Gustatory Stimulation}

Litters were separated from the mother over night before the day of the experiment (21:00 to 09:00 h) and deprived of food and water. On the day of the experiment females were removed and males were placed in a plexiglass $(33 \times 23 \times 15 \mathrm{~cm})$ cage in a quiet room, and the gustatory stimulation was performed between 09:00 and 10:30 h. Thereafter, they were randomly divided into controls and experimental groups, each consisting of four rats $(n=4)$ for each age and experimental condition.

We use two control groups, one that is isolated from the mother until sacrifice (WS), and another group that was stimulated with distilled water (W). The experimental groups were animals receiving one of the following gustatory stimuli: $0.003 \mathrm{M}$ quinine hydrochloride $(\mathrm{QHCl})$, $0.1 \mathrm{M}$ sodium chloride $(\mathrm{NaCl})$, and $0.1 \mathrm{M}$ sucrose $(\mathrm{S})$. The gustatory cues were manually administered with the aid of an automatic pipette; rats were given 5 stimula- tions, each with $10 \mu \mathrm{l}$ of a particular taste solution, at 5-min intervals over a 20-min period (Figure 1).

\subsection{Immunohistochemical Staining}

After the intraoral stimulation, each rat remained in the chamber and was sacrificed 90 min after the first stimulation. Preliminary experiments showed that the combination of a 12-h isolation from the mother followed by sacrifice and perfusion after 90 min was optimal: It resulted in a low basal level of fos expression in the controls or detectable induction of fos in stimulated animals. Just before sacrifice, rats were deeply anesthetized with urethane (115 mg/100g b.w., i. p.) and perfused via the ascending aorta with saline, followed by $4 \%$ paraformaldehyde ( $\mathrm{pH} \mathrm{9.5,} 10^{\circ} \mathrm{C}$ ). Brains were removed, post-fixed for $24 \mathrm{~h}$, and cryoprotected in 10\% sucrose-phosphate buffer overnight at $4^{\circ} \mathrm{C}$. The brainstem was cut in coronal sections of $50 \mu \mathrm{m}$ on a freezing microtome; three series across the length of the brainstem were collected and stored in cryoprotectant (30\% ethylene glycol and 20\% glycerol in $0.05 \mathrm{M}$ sodium phosphate buffer) at $-20^{\circ} \mathrm{C}$ until histochemical processing.

Fos-like immunoreactivity (FLI) was detected using a conventional avidin-biotin-immunoperoxidase technique [26]. To minimize variations in the immunohistochemical processing, tissue from animals in different groups was processed simultaneously. Processing was performed on free-floating sections. As negative control, some tissue was separated prior to the primary antibody incubation and placed into the PBS-0.3\% Triton X-100 solution with no primary antibody. Sections were pretreated with hydrogen peroxide for $10 \mathrm{~min}$ to quench endogenous peroxidase activity, followed by 2 rinses in PBS and then incubated by $10 \mathrm{~min}$ in $1.0 \%$ sodium borohydride to reduce free aldehydes. Sections were then incubated with polyclonal antiserum raised against the N-terminal fragment of human fos protein, Santa Cruz Biotechnologies, CA, USA, diluted 1:5000 into the PBS-0.3\% Triton X-100 with $400 \mu$ l of normal goat serum solution at $4^{\circ} \mathrm{C}$ for $48 \mathrm{~h}$. The sections were rinsed,

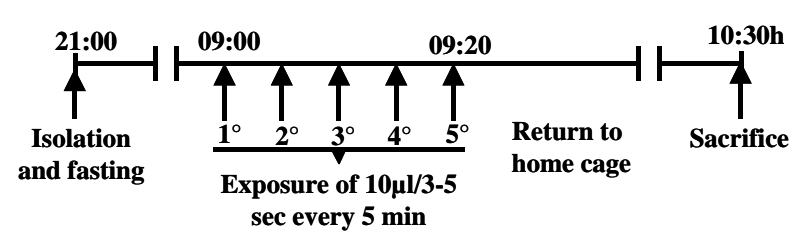

Experimental Groups:
0.003 M QHCl
0.1 M sucrose
0.1 M NaCl
Water
Without stimulation

Figure 1. Schedule of gustatory stimulation. 
incubated with biotinylated goat anti-rabbit secondary antiserum (Vector Laboratories, Inc. Burlingame, CA) diluted 1:300 with PBS-0.3\% Triton X-100 at room temperature for $1 \mathrm{~h}$ and processed using the standard biotin avidin-peroxidase kit (Vectastain Elite ABC kit, Vector Laboratories, Burlingame, CA, USA) for $1.5 \mathrm{~h}$ at room temperature. Sections were rinsed four times in PBS and reacted with nickel chloride-3,3'-diaminoben-zidine and $0.0003 \%$ hydrogen peroxide for 7 min (DAB kit, Vector Laboratories, Burlingame, Ca, USA), sections were rinsed and mounted onto gelatin-coated slides and cover slipped using permount. Control experiments, in which the primary antiserum was preabsorbed with antigen overnight, showed no specific nuclear labeling in tissue from control or experimental animals

\subsection{Counting of FLI Neurons in the rNST and Statistical Analysis}

In order to compare differences in the number of cells activated by taste stimuli, we analyzed distribution of fos positive cells located in the medial, intermediate and lateral area of the NST in coronal planes. For quantification, the number of cells per section at four rostro-caudal levels was used for average results. In a 5-day old rat pup, all brainstem nuclei are identifiable in their final locations, and structurally are very similar to those of the adult rat and can be recognized on the basis of similarity with the adult equivalent [27]. The four rostro-caudal levels were: in the rNST, Bregma $-12.30 \mathrm{~mm}$ level 1 (L1) and Bregma $-13.24 \mathrm{~mm}$ (L2), in the intermediate NST, Bregma $-13.80 \mathrm{~mm}$ (L3) and caudal NST, Bregma $-14.30 \mathrm{~mm}$ (L4) in reference to the Bregma from the adult rat brain atlas [28]. In present study, we counted at $20 \times$ only fospositive nuclei seen as dark, round or oval structures. Each slide was assigned a random number to ensure that observations were blind with respect to age and taste stimuli of subjects. The FLI-positive cells in each section were counted, and the mean number of cells for each region was analyzed.

\subsection{Statistical Analysis}

Because there were not significant differences between the WS and W groups, only the water (W) group was used as control for all subsequent statistical comparisons. For the analyses we summed across all four levels, using a three-way ANOVA with stimulus as one factor, age as second factor, and either antero-caudal level or medial-lateral subfield as third factor. Separate, two-way ANOVAs were calculated for each level (summed across subfields) or each subfield (summed across levels) as one repeated measures factor and age as a second factor. Post hoc comparisons at each developmental age were made by using the Fisher Least Square Differences (LSD) test.
The threshold for significance was set at $\mathrm{p}<0.05$.

\section{Results}

Analysis of the rNST coronal sections from the preweaned rats subjected to the experimental conditions proved that isolation from the mother resulted in a basal level of c-fos expression at all ages studied. After establishing this basal level, the gustatory stimulation induced a significant response in the rNST, as determined by the FLI. The pups that were stimulated with QHCl and S showed a clear and more intense FLI in the rNST, but $\mathrm{W}$ and $\mathrm{NaCl}$ caused a minor activational response (Figure 2).

\subsection{FLI Distribution in the Different Rostro-Caudal Levels of the rNST in Response to Specific Gustatory Cues}

Statistical comparisons in the number of FLI-positive nuclei in quinine-stimulated rats showed significant differences associated with age, $F(2,9)=86.14, \mathrm{p}<0.001$, and rNST levels, $\mathrm{F}(3,27)=9.98, \mathrm{p}<0.001$, without interaction. Post hoc comparisons at different levels and ages are displayed in Figure 3. Throughout the study, quinine showed consistently higher values in FLI compared to the other taste stimuli with a peak on day 15 (Figure 3).

The amount of FLI in the S group exhibited significant differences associated with age, $\mathrm{F}(2,9)=31.4, \mathrm{p}<0.001$ and the rNST level, $F(3,27)=31.5, p<0.001$, with no interaction between factors. Post hoc comparisons at
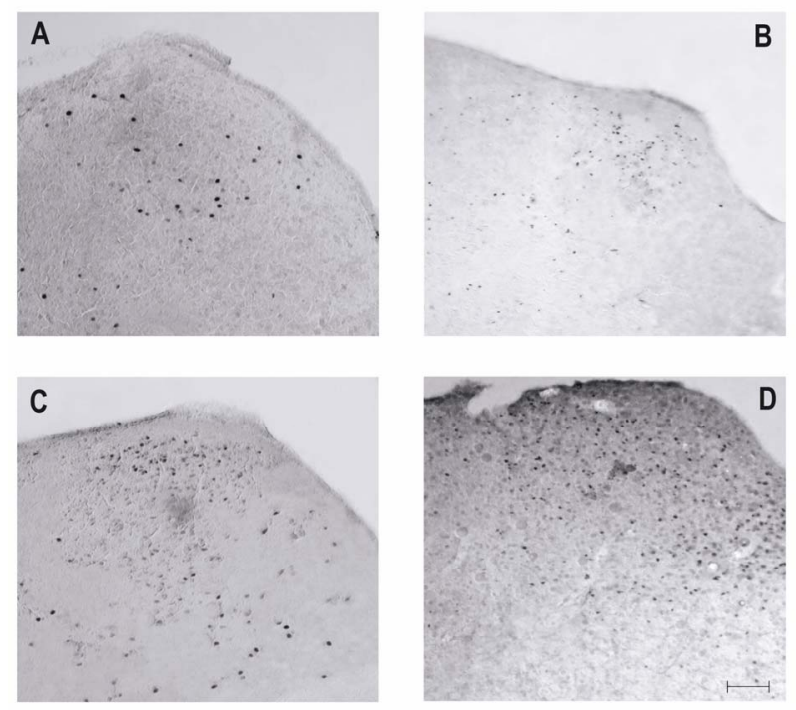

Figure 2. Photomicrographs of coronal sections of the rNST at level of Bregma $-13.24 \mathrm{~mm}(\mathrm{~L} 2)$ in the current study. (A) Water and (B) $\mathrm{NaCl}$ stimulation was associated with weak FLI. By contrast, after (C) sucrose, and (D) quinine stimulation FLI was more intense. Calibration $200 \mu \mathrm{m}$. 
each developmental age are shown in Figure 3. FLI in the S group gradually declined along the rNST levels and was lower than in the quinine group but higher than in groups exposed to the other taste cues (Figure 3).

FLI in the $\mathrm{NaCl}$ and $\mathrm{W}$ groups were only modified by the rNST levels, $\mathrm{F}(3,27)=5.97, \mathrm{p}<0.001$, and $\mathrm{F}(3,27)=$ $3.03, \mathrm{p}<0.046$ respectively with no interaction between factors. Post hoc comparisons at different developmental ages are presented in Figure 3. FLI in the $\mathrm{NaCl}$ and $\mathrm{W}$ groups at different rNST levels and ages was lower than in the quinine and $\mathrm{S}$ groups (Figure 3 ).

\subsection{FLI Distribution in the Different Medio-Lateral Subfields of the rNST in Response to Specific Gustatory Cues}

Statistical comparisons of the amount of FLI in qui- nine-stimulated rats showed significant differences associated with age, $\mathrm{F}(2,44)=24.46, \mathrm{p}<0.001$ and rNST subfield, $\mathrm{F}(2,88)=125.30, \mathrm{p}<0.001$. Additionally, a significant interaction between age and rNST subfield, $\mathrm{F}(4,88)=14.80, \mathrm{p}<0.001$, was obtained. Post hoc comparisons at each day of the study are shown in Figure 4. The amount of FLI was generally higher with quinine compared to the other gustatory cues, and it decreased toward the lateral subfield.

The amount of FLI in the sucrose group was not affected by age but was significantly different between rNST subfields, $F(2,88)=34.55$, $\mathrm{p} \leq 0.001$. Furthermore, there was a significant interaction between age and rNST subfield, $F(4,88)=7.4, p<0.02$. Post hoc comparisons over the course of the study are displayed in Figure 4. Sucrose increased FLI in the medial and lateral rNST

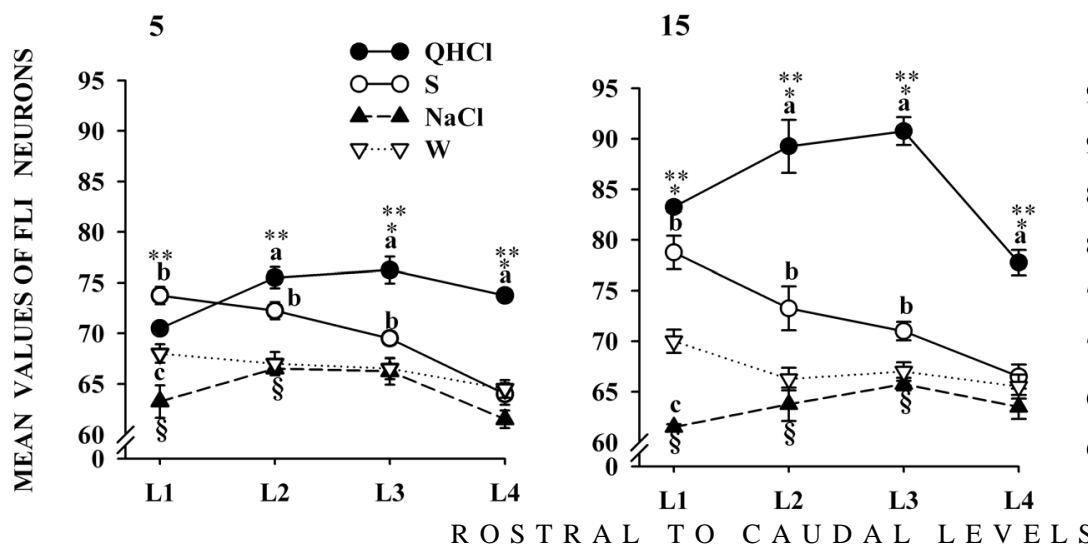

25

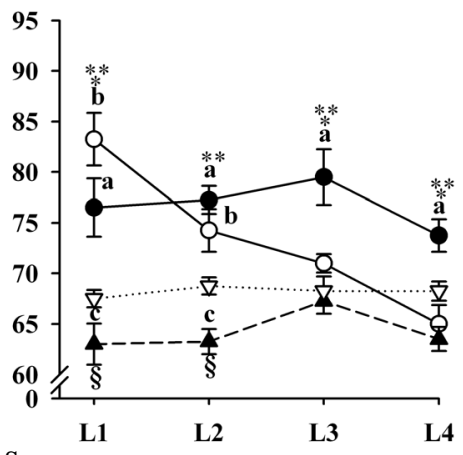

Figure 3. Mean number of FLI neurons in different levels of the rNST. Neurons are summed across subnuclei of animals in the four stimulation conditions. Post hoc analysis indicate that both quinine and sucrose resulted in significantly more FLI than the other groups $(p<0.05$ indicated by a and $b)$. But the increased FLI elicited by NaCl compared with water was only significant in some cases $(\mathrm{p}<0.05)$. QHCl: quinine; S: sucrose; W: water.

5

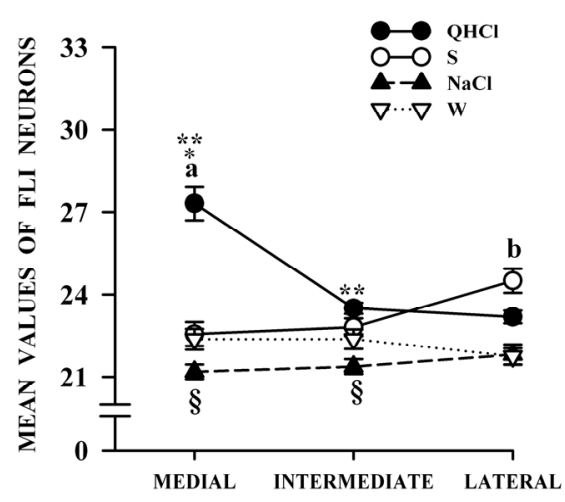

15

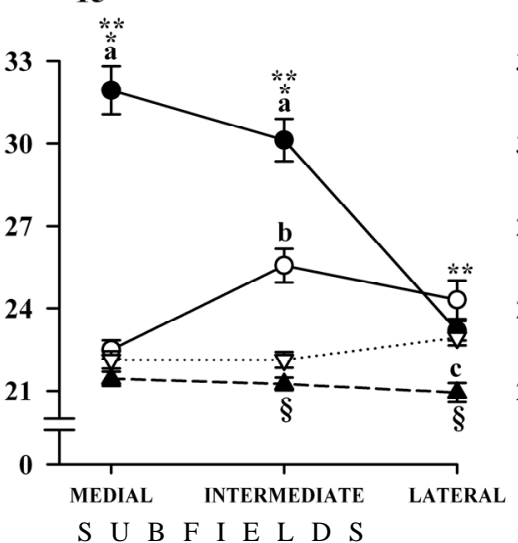

25

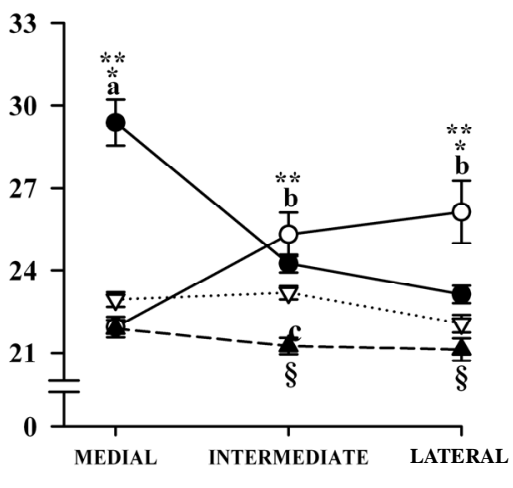

Figure 4. Mean number of FLI neurons in different subfields of the rNST. Neurons are summed across levels of animals in the four stimulation conditions. Post hoc analysis indicate that both quinine induce more FLI in medial zone in contrast sucrose resulted in significantly more FLI in lateral subnuclei at 25 days of age $(p<0.05$ indicated by and a and $b)$. But the increased FLI elicited by NaCl compared with water was only significant in some cases $(p<0.05)$. QHCl: quinine; S: sucrose; W: water. 
subfields more than the other taste stimuli.

Statistical comparisons showed that the number of FLI-positive cells in the NaCl-treated rats was not affected by age or subfield, nor was a significant interaction between factors observed. Statistical comparisons of the number of FLI-positive cells in the $\mathrm{W}$ group show no significant differences with age or subfield. However, the interaction between gustatory cue and rNST subfield, $\mathrm{F}(4,88)=3.67, \mathrm{p}<0.01$, was significant. Post hoc comparisons over the course of the study are shown in Figure 4.

\section{Discusion}

Our data indicate a clear FLI in the rat rNST as early as P5, suggesting that the neuronal connections between rNST neurons and taste receptors may not be complete but are operating at P5. In this regard the FLI of this gustatory area appears to be very similar to the FLI detected in the newborn olfactory bulb which possibly underlies basic newborn functions such as food intake, mother recognition, and the establishment of early associative learning with taste stimuli of the nest environment as a mechanism to gain survival experience $[2,29]$. This possibility is supported by previous electrophysiological, morphological, and behavioral observations of gustatory responses in NST area [18-21,30-36] suggesting that they are part of ancient, complex brainstem mechanisms modulating cardio-respiratory rhythms and food-intake in several species [37].

Quinine elicits intense FLI primarily in the medial subfield. Following oral quinine exposure found that newborn pups exhibited intense mouth opening and reduced lip licking and face-pulling [38]. Thus, quinine stimulation activates not only a small neuronal subpopulation of the rNST associated with the taste sensation, but also a neuronal subset related to oromotor responses [39]. Similar studies performed in the adult rat indicated that quinine evoked FLI segregated within the medial subfields of the rNST $[17,24,25,39,40]$.

The current results also indicate that FLI elicited by taste stimuli are primarily related to the oral gustatory receptors rather than to the digestive tract visceral recaptors activated after ingestion. Yamamoto and Sawa, 2000 [41] recently reported that the intragastric infusion of 1 $\mathrm{mM}$ quinine induced FLI in the visceral cNST area at a site where the intraoral infusion of quinine fails to induce FLI. Although quinine oral stimulation may directly activate gastrointestinal receptors and their afferents, we recognize that the FLI at the most caudal level of the rNST examined here may represent a combination of visceral and gustatory effects, since these regions receive significant input from the IX and X cranial nerves [40]. Nevertheless, this suggestion requires further investiga- tion in order to understand how different gustatory recaption areas combine their ascending information to integrate the sensory and hedonic aspects of the gustatory signals.

Another possible explanation for the quinine effects seen here is the contribution of the tactile stimulation provoked by the liquid volume introduced into the oral cavity during the stimulation that was perhaps superimposed on the gustatory stimulus. Giving support to this possibility are the anatomical convergence of the IX and $\mathrm{X}$ cranial afferents in the rNST, the increased rNST neuronal electrical discharges following the oral mechanical stimulation, and the observation here of slight FLI in the rNST elicited by water stimulation [15,16,25,42-44]. However, the role of the tactile influence of liquid on the oral receptors is apparently negligible. Our data show that sucrose generally elicits less FLI than quinine but more than $\mathrm{NaCl}$ and $\mathrm{W}$ at all ages; the FLI gradually declines along the rostro-caudal levels of the rNST except on day P5, but increases with age along the medial-lateral subfields. This profile of sucrose is in line with the higher preference of the newborn for sucrose, compared to $\mathrm{NaCl}$ and Water, suggesting that in the rNST, the threshold for neuronal activation by sucrose is reduced during the pre-weaning period [45]. The FLI elicited by $\mathrm{NaCl}$ in the rNST at various ages was similar to that elicited in the $\mathrm{W}$ subjects. The $\mathrm{NaCl}$ concentration used was similar to that in amniotic fluid, an important component of the fetal environment [46]. Thus, the $\mathrm{W}$ and $\mathrm{NaCl}$ cues used here to elicit FLI in the rNST might cause a lowintensity activation of gustatory receptors.

The fos immunohistochemistry technique has some limitations, one of which is that stimulus-induced c-fos expression occurs only in those neurons that generate a sufficient amount of neuronal activity. Accordingly, very high stimulus intensities must typically be used to achieve measurable FLI. Moreover, in some brain regions, neurons remain fos-immunonegative regardless of the strength or duration of the stimulus [45]. The reason for this is still unclear, but may be related to biochemical messengers that regulate neuronal c-fos activation [47] or to the preferential expression of transcription factors other than c-fos [48-50]. It is possible that cells were activated by other tastants here used that escaped FLI detection.

In the rNST these FLI distribution are taste specific, and the spatial location apparently changes during postnatal development. The findings presented here suggest that these subfields of the rNST may play an important role throughout the first two weeks of life, but the details of their development are unknown. The synaptogenesis of sensory afferents that originate in the taste buds, the projections to the reticular formation, gustatory cortex, amygdala, and other areas, as well as the metabolic ma- 
turation of the brainstem, and the levels of inhibitory and stimulatory neuromodulators and neurotransmitters may contribute to the postnatal maturation and function of the rNST. Age is another important factor that modulates postnatal changes in the number of gustatory-induced FLI-positive neurons from P5 to P15, a time when the gustatory receptors are still maturing. These results are in agreement with previous studies performed in other brain structures that continue to develop during early postnatal life [51,52].

Taken together, the findings reported here suggest that the gustatory-specific patterns of activity in the rNST are established during the perinatal period and do not undergo substantial remodeling during the neonatal period. However, further studies are necessary in order to understand the plastic properties of this brainstem substrate that is fundamental for newborn survival.

\section{Conclusion}

In conclusion, difference in the FLI expression between sucrose and quinine was demonstrated and the middle and lateral regions respectively showed higher FLI expression than water or $\mathrm{NaCl}$ in rats as early as 5 days of age and change during early development. The results provide morphological evidence for perceptional and discrimination of taste signaling in the NST.

\section{Acknowledgements}

Partly supported by DGAPA/UNAM, IN207-310, IN207307, CONACyT No. 171332 and DGAPA/UNAM No. 503001915 scholarship to L.R. We thank Dr. D. Pless for editorial assistance, N. Hernandez for help with the images, and to the UNAM Biomedical Sciences, PhD program.

\section{REFERENCES}

[1] J. M. Cernoch and R. H. Porter, "Recognition of Maternal Axillary Odors by Infants,” Child Development, Vol. 56, No. 6, 1985, pp. 1593-1598. doi:10.2307/1130478

[2] S. Moriceau and R. M. Sullivan, "Maternal Presence Serves as a Switch between Learning Fear and Attraction in Infancy,” Nature Neuroscience, Vol. 9, No. 8, 2006, pp. 1004-1006. doi:10.1038/nn1733

[3] I. Farbman "Electron Microscope Study of the Developing Taste Bud in Rat Fungiform Papilla,” Developmental Biology, Vol. 11, No. 1, 1965, pp. 110-135. doi:10.1016/0012-1606(65)90040-0

[4] M. Mistreta, "Topographic and Histological Study of the Developing Rat Tongue, Palate and Taste Buds,” In: J. F. Bosma, Ed., Third Symposium on Oral Sensations and Perception, Charles C Thomas, Springfield, 1972, pp. 163187.

[5] J. P. Mbiene and A. I. Farbman, "Evidence for Stimulus
Access to Taste Cells and Nerves during Development: An Electron Microscopic Study,” Microscopy Research and Technique, Vol. 26, No. 2, 1993, pp. 94-105. doi:10.1002/jemt.1070260203

[6] M. A. Hosley and B. Oskley, "Postnatal Development of the Vallate Papilla and Taste Buds in Rats," The Anatomical Record, Vol. 218, No. 2, 1987, pp. 216-222. doi:10.1002/ar.1092180217

[7] R. F. Krimm and D. L. Hill, "Innervation of Single Fungiform Taste Buds during Development in Rat," The Journal of Comparative Neurology, Vol. 398, No. 1, 1998, pp. 13-24.

doi:10.1002/(SICI)1096-9861(19980817)398:1<13::AIDCNE2>3.0.CO;2-C

[8] P. S. Lasiter, D. M. Wong and D. L Kachele, "Postnatal Development of the Rostral Solitary Nucleus in Rat: Dendritic Morphology and Mitochondrial Enzyme Activity,” Brain Research Bulletin, Vol. 22, No. 2, 1989, pp. 313-321. doi:10.1016/0361-9230(89)90059-2

[9] P. S. Lasiter and D. L. Kachele, "Elevated NADH-Dehydrogenase Activity Characterizes the Rostral Gustatory Zone of the Solitary Nucleus in Rat,” Brain Research Bulletin, 1989, Vol. 22, No. 4, pp. 777-781. doi:10.1016/0361-9230(89)90099-3

[10] L. Rubio, C. Torrero, M. Regalado and M. Salas, “Alterations in the Solitary Tract Nucleus of the Rat Following Perinatal Food Restriction and Subsequent Nutritional Rehabilitation,” Nutritional Neuroscience, Vol. 7, No. 56, 2004, pp. 291-300. doi:10.1080/10284150400019922

[11] H. Bao, R. M. Bradley and C. M. Mistretta, "Development of Intrinsic Electrophysiological Properties in Neurons from the Gustatory Region of Rat Nucleus of Solitary Tract,” Developmental Brain Research, Vol. 86, No. 1-2, 1995, pp. 143-154. doi:10.1016/0165-3806(95)00020-E

[12] M. Mistretta and S. Labyak, "Maturation of Neuron Types in Nucleus of Solitary Tract Associated with Functional Convergence during Development of Taste Circuits,” The Journal of Comparative Neurology, Vol. 345, No. 3, 1994, pp. 359-376. doi:10.1002/cne.903450304

[13] W. E. Renehan, J. Massey, Z. Jin, X. Zhang, Z. Liu and L. Schweitzer, "Developmental Changes in the Dendritic Architecture of Salt-Sensitive Neurons in the Nucleus of the Solitary Tract," Developmental Brain Research, Vol. 102, No. 2, 1997, pp. 231-246. doi:10.1016/S0165-3806(97)00104-1

[14] A. Vincent and F. Tell, "Postnatal Development of Rat Nucleus Tractus Solitarius Neurons: Morphological and Electrophysiological Evidence,” Neuroscience, Vol. 93, No. 1, 1999, pp. 293-305. doi:10.1016/S0306-4522(99)00109-8

[15] M. I. Harrer and S. P. Travers, “Topographic Organization of Fos-Like Immunoreactivity in the Rostral Nucleus of the Solitary Tract Evoked by Gustatory Stimulation with Sucrose and Quinine,” Brain Research, Vol. 11, No. 1-2, 1996, pp. 125-137. doi:10.1016/0006-8993(95)01410-1 
[16] S. P. Travers and H. Hu, "Extranuclear Projections of rNST Neurons Expressing Gustatory-Elicited Fos,” The Journal of Comparative Neurology, Vol. 427, No. 1, 2000, pp. 124-138.

doi:10.1002/1096-9861(20001106)427:1<124::AID-CNE 8>3.0.CO;2-2

[17] S. P. Travers, "Quinine and Citric Acid Elicit Distinctive Fos-Like Immunoreactivity in the Nucleus of the Solitary Tract," American Journal of Physiology, Vol. 282, No. 6, 2002, pp. 1798-1810.

[18] J. R. Ganchrow, J. E. Steiner and S. Canetto, "Behavioral Displays to Gustatory Stimuli in Newborn Rat Pups," Developmental Psychobiology, Vol. 19, No. 3, 1986, pp. 163-174. doi:10.1002/dev.420190303

[19] G. J. Shwartz and H. J. Grill, "Comparing Taste-Elicited Behaviors in Adult and Neonatal Rats,” Appetite, Vol. 6, No. 4, 1985, pp. 373-386. doi:10.1016/S0195-6663(85)80005-2

[20] B. Johanson and E. G. Shapiro, "Intake and Behavioral Responsiveness to Taste Stimuli in Infant Rats from 1 to 15 Days of Age,” Developmental Psychobiology, Vol. 19, No. 6, 1986, pp. 593-606. doi:10.1002/dev.420190610

[21] W. G. Hall and T. E. Bryan, "The Ontogeny of Feeding in Rats: IV. Taste Development as Measured by Intake and Behavioral Responses to Oral Infusions of Sucrose and Quinine," Journal of Comparative and Physiological Psychology, Vol. 95, No. 2, 1981, pp. 240-251. doi: $10.1037 / \mathrm{h} 0077771$

[22] M. Sagar, F. R. Sharp and T. Currant, "Expression of cFos Protein in Brain: Metabolic Mapping at the Cellular Level,” Science, Vol. 240, No. 4857, 1988, pp. 13281331. doi:10.1126/science.3131879

[23] L. A. DiNardo and J. B. Travers, "Distribution of FosLike Immunoreactivity in the Medullary Reticular Formation of the Rat after Gustatory Elicited Ingestion and rejection Behaviors," The Journal of Neuroscience, Vol. 17, No. 10, 1997, pp. 3826-3839.

[24] C. T. King, S. P. Travers, N. E. Rowland, M. Garcea and A. C. Spector, "Glossopharyngeal Nerve Transection Eliminates Quinine-Stimulated Fos-Like Immunoreactivity in the Nucleus of the Solitary Tract: Implications for a Functional Topography of Gustatory Nerve Input in Rats,” The Journal of Neuroscience, Vol. 19, No. 8, 1999, pp. 3107-3121.

[25] S. M. Altshuler, X. Bao, D. Bieger, D. A. Hopkins and R. R. Miselis, "Viscerotopic Representation of the Upper Alimentary Tract in the Rat: Sensory Ganglia and Nuclei of the Solitary and Spinal Trigeminal Tracts," The Journal of Comparative Neurology, Vol. 283, No. 2, 1989, pp. 248-268. doi:10.1002/cne.902830207

[26] T. Morales, L. Aguilar, E. Ramos, F. Mena and C. Morgan, "Fos Expression Induced by Milk Ingestion in the Caudal Brainstem of Neonatal Rats,” Brain Research, Vol. 1241, No. 19, 2008, pp. 76-83. doi:10.1016/j.brainres.2008.09.022

[27] G. Paxinos, K. W. S. Ashwell and I. Tork, "Atlas of the Developing Rat Nervous System,” 2nd Edition, Academic Press, Waltham, 1994.

[28] G. Paxinos and C. Watson, "The Rat Brain in Stereotaxic
Coordinates,” Academic Press, Waltham, 1997.

[29] S. S. Miller and N. E. Spear, "Olfactory Learning in the Rat Neonate Soon after Birth,” Developmental Psychobiology, Vol. 50, No. 6, 2008, pp. 554-565. doi:10.1002/dev.20318

[30] D. Hill and C. R. Almil, "Ontogeny of Chorda Tympani Nerve Responses to Gustatory Stimuli in the Rat," Brain Research, Vol. 197, No. 1, 1980, pp. 27-38. doi:10.1016/0006-8993(80)90432-1

[31] D. Hill, R. M. Bradley and C. M. Mistretta, "Development of Taste Responses in the Rat Nucleus of Solitary Tract,” Journal of Neurophysiology, Vol. 50, No. 4, 1983, pp. 879-895.

[32] D. M. Wong and D. L. Kachele, "Postnatal Development of the Rostral Solitary Nucleus in Rat: Dendritic Morphology and Mitochondrial Enzyme Activity,” Brain Research Bulletin, Vol. 22, No. 2, 1989, pp. 313-321. doi:10.1016/0361-9230(89)90059-2

[33] P. S. Lasiter, "Postnatal Development of Gustatory Recipient Zones within the Nucleus of the Solitary Tract," Brain Research Bulletin, Vol. 28, No. 5, 1992, pp. 667677. doi:10.1016/0361-9230(92)90245-S

[34] A. K. Thaw, S. Frakmann and D. L. Hill, "Behavioral Taste Responses of Developmentally NaCl-Restricted Rats to Various Concentrations of NaCl," Behavioral Neuroscience, Vol. 114, No. 2, 2000, pp. 437-441. doi:10.1037/0735-7044.114.2.437

[35] O. May and D. Hill, “Gustatory Terminal Field Organization and Developmental Plasticity in the Nucleus of the Solitary Tract Revealed through Triple-Fluorescence Labeling," The Journal of Comparative Neurology, Vol. 497, No. 4, 2006, pp. 658-669. doi:10.1002/cne.21023

[36] J. Mangold and D. Hill, "Postnatal Reorganization of Primary Afferent Terminal Fields in the Rat Gustatory Brainstem Is Determined by Prenatal Dietary History,” The Journal of Comparative Neurology, Vol. 509, No. 6, 2008, pp. 594-607. doi:10.1002/cne.21760

[37] J. E. Steiner and D. Glaser, "Differential Behavioural Responses to Taste Stimuli in Non-Human Primates," Journal of Human Evolution, Vol. 13, No. 8, 1984, pp. 709-723. doi:10.1016/S0047-2484(84)80021-4

[38] J. E. Steiner, "Human Facial Expressions in Response to Taste and Smell Stimulation,” In: R. W. Hayne and L. P. Lipsitt, Eds., Advance in Child Development and Behavior, Academic Press, Inc., New York, 1979, pp. 257-293.

[39] S. P. Travers and J. B. Travers, "Reflex Topography in the Nucleus of the Solitary Tract," Chemical Senses, Vol. 30, No. 1, 2005, pp. 180-181. doi:10.1093/chemse/bjh173

[40] M. C. Whitehead, "Neuroanatomy of the Gustatory System,” Gerodontics, Vol. 5, No. 5, 1988, pp. 239-243.

[41] T. Hanamori and N. Oshiko, "Cardiovascular Responses to Gustatory and Mechanical Stimulation of the Nasopharynx in Rats,” Brain Research, Vol. 619, No. 1-2, 1993, pp. 214-222. doi:10.1016/0006-8993(93)91614-X

[42] T. Yamamoto and K. Sawa, "c-Fos like Immunoreactivity in the Brainstem Following Intraoral and Intragastrica Infusion of Chemical Solutions in Rats”, Brain Research, Vol. 866, No. 1-2, 2000, pp. 144-151. 


\section{doi:10.1016/S0006-8993(00)02242-3}

[43] H. Ogawa, T. Imoto and T. Hayama, "Responsiveness of Solitario-Parabrachial Relay Neurons to Taste and Mechanical Stimulation Applied to the Oral Cavity in Rats,” Experimental Brain Research, Vol. 54, No. 2, 1984, pp. 349-358.

[44] S. P. Travers and R. Norgren, "Organization of Orosensory Responses in the Nucleus of the Solitary Tract of the Rat,” Journal of Neurophysiology, Vol. 73, No. 6, 1995, pp. 2144-2162.

[45] E. Erkadius, T. O. Morgan and R. Di Nico, "Aminiotic Fluid Composition and Fetal and Placental Growth Rates in Genetically Hypertensive and Normotensive Rats," Reproduction, Fertility and Development, Vol. 7, No. 6, 1995, pp. 1563-1567. doi:10.1071/RD9951563

[46] R. Hudson and H. Distel, "The Flavour of Life: Perinatal Development of Odour and Taste Preferences," Schweizerische Medizinische Wochenschrift, Vol. 129, No. 5, 1999, pp. 176-181.

[47] M. Dragunow and R. Fall, "The Use of c-Fos as a Metabolic Marker in Neuronal Pathway Tracing,” Journal of Neuroscience Methods, Vol. 29, No. 3, 1989, pp. 261265. doi:10.1016/0165-0270(89)90150-7
[48] M. Sheng and M. E. Greenberg, "The Regulation and Function of c-Fos and Other Immediate Early Genes in the Nervous System,” Neuron, Vol. 4, No. 4, 1990, pp. 477- 485. doi:10.1016/0896-6273(90)90106-P

[49] J. I. Morgan and T. Currant, "Stimulus-Transcription Coupling in the Nervous System: Involvement of the Inducible Proto-Oncogenes Fos and Jun,” Annual Review Neuroscience, Vol. 14, No. 1, 1991, pp. 421-451. doi:10.1146/annurev.ne.14.030191.002225

[50] L. Kaczmarek and A. Chaudhuri, "Sensory Regulation of Immediate-Early Gene Expression in Mammalian Visual Cortex: Implications for Functional Mapping and Neural Plasticity,” Brain Research Reviews, Vol. 23, No. 3, 1997, pp. 237-256. doi:10.1016/S0165-0173(97)00005-2

[51] R. Wickstrom, T. Hokfelt and H. Lagererantz, "Development of $\mathrm{CO}_{2}$-Responses in the Early Newborn Period in Rat," Respiratory Physiology \& Neurobiology, Vol. 132, No. 2, 2002, pp. 145-158. doi:10.1016/S1569-9048(02)00076-9

[52] K. M. Guthrie and C. Gall, “Anatomic Mapping of Neuronal Odor Responses in the Developing Rat Olfactory Bulb,” The Journal of Comparative Neurology, Vol. 455, No. 1, 2003, pp. 56-71. doi:10.1002/cne.10452 